\title{
Zum geschlechtergerechten Sprachgebrauch am Beispiel deutscher und polnischer Stellenausschreibungen
}

\author{
Marek Cieszkowski (Bydgoszcz)
}

\begin{abstract}
The subject of consideration is the issue of using terms for professions which linguistically discriminate women or men in German and Polish job offers. Problems connected with the above were presented in the context of achievements of feminist linguistics in Germany and comprise a critical view of language asymmetries in Polish as well as the way of presenting it in Polish scientific discourses.
\end{abstract}

\section{$1 \quad$ Einleitung}

In der folgenden Darstellung wird das Problemfeld der geschlechtergerechten Ausdrucksweise am Beispiel einer deutsch-polnischen Korpusdatei untersucht. Zum einen erfolgt eine kontrastive Bestandsaufnahme deutscher und polnischer Sprachdaten, zum anderen werden dabei aktuelle Probleme der Bildung und Verwendung von femininen Formen im Polnischen zur Diskussion ${ }^{1}$ gestellt, die im öffentlichen Diskurs zielbewusst und immer häufiger gebraucht werden. Die polonistische Linguistik hat sich diesem Phänomen mit großem Interesse zugewandt, auch wenn sie an diesen Fragenkomplex immer noch mit gewissen Bedenken herangeht. Das lässt sich u. a. an verschiedenen sprachlichen Distanzierungstechniken der ReferentInnen beobachten, wenn über verschiedene Methoden und erbrachte Forschungsergebnisse in Zusammenhang mit dem Feminismus diskutiert wird. Bevor ich jedoch zur Auswertung und Interpretation des erfassten Sprachkorpus übergehe, möchte ich mich zunächst mit einigen Begriffen auseinandersetzen, die einen theoretischen Hintergrund bilden und praktikable Verallgemeinerungen begründen helfen. Ausgegangen wird von den Grundprinzipien der feministischen Linguistik, die sich als neue wissenschaftliche Disziplin bereits in den 80er-Jahren in der BRD etabliert hat, speziell bei polnischen AutorInnen thematisiert wurde, eng mit politischen Entwicklungen zusammenhängt und im Kontext sozial- und sprachpolitischer Transformationsprozesse der (west)europäischen Gesellschaften gesehen wird.

1 Aus formalen und thematischen Gründen können im Rahmen dieses Beitrags nur ausgewählte Aspekte behandelt werden, die mit dem Aufkommen von neuen femininen Formen im Polnischen zusammenhängen und sich durch den verändernden, mediengestützten Sprachgebrauch in Polen dokumentieren lassen. 


\section{Genderorientierte Sichtweise der modernen Polonistik}

In Polen sind in den letzten Jahrzehnten einige Monographien, Sammelbände sowie andere Publikationen erschienen, u. a. Einzelbeiträge und Artikel in wissenschaftlichen Zeitschriften (vgl. Literaturverzeichnis), in denen man zwar den roten Faden einer linguistischen, genderorientierten Sichtweise erkennen kann, aber all diese Beiträge zeugen von einem sich erst allmählich anbahnenden Diskurs und ermöglichen höchstens eine modulare und methodologisch uneinheitliche Explorierung des genderbeziehbaren Paradigmas. Diskutiert werden nicht das thematische Spektrum der Gender Studies und die Verankerung der linguistischen Genderproblematik im interdisziplinären Umfeld, was im Nachhinein zur Herauskristallisierung einer neuen Teildisziplin beitragen könnte, sondern das gesamte Problem wird meines Erachtens aus einer glass-ceiling-effect-Perspektive ohne einschneidende sprachpolitische Konsequenzen angegangen, von denen in der polnischen Linguistik bewusst Abstand genommen wird. Im weiteren Teil des Beitrags komme ich auf diese Konsequenzen noch einmal zurück.

Untersuchungen zum Verhältnis von Sprache und Geschlecht haben in Polen eine ziemlich lange Tradition; ein Blick in die Forschung lässt erkennen, dass erste Aufsätze zu maskulinen und femininen Berufs- und Personenbezeichnungen bereits zu Beginn des 20. Jh. erschienen sind (cf. Belczyk-Kohl 2013: 22-24). Die Problematik wurde mit unterschiedlicher Intensität auch in den 50er- und 60er-Jahren fortgesetzt. Zuletzt rückte sie in den 80-er-Jahren in das Blickfeld der polonistischen Linguistik (cf. Handke 1986; 1989) und wird konsequenterweise bis heute thematisiert. All diesen Perioden ist jedoch gemeinsam, dass maskuline und feminine Berufsbezeichnungen sowie Titel meist aus der sprachpflegerischen Sicht bearbeitet und lediglich im Hinblick auf die sprachliche Norm oder morphologisch-syntaktische Besonderheiten des Polnischen exemplifiziert werden. Nichtsdestoweniger bekunden weitere Publikationen von Handke (cf. Anusiewicz/Handke 1994; Handke 1990; 1992; 1994b; 1995) eine teilweise neue, eigenständige Forschungsperspektive mit gendergerechter Haltung, auch wenn sie sich gleich in der Anfangsphase vom Feminismus ${ }^{2}$ distanzieren will, ihr wissenschaftliches Betätigungsfeld auf die Interpretation des sprachlichen Materials zurückführt und ihre Arbeiten als soziolinguistisch bezeichnet (cf. Handke 1994a: 11-12). In diesem Zusammenhang steht auch eine Reihe von Veröffentlichungen, die u. a. die Erkenntnisse der Lubliner Schule der PolonistInnen belegen: Karwatowska (2004) und Karwatowska/Szpyra-Kozłowska (2005) nutzen nur teilweise feministische Methodologien und Methoden, indem sie kritisch über das Phänomen der sprachlichen Asymmetrien im Polnischen diskutieren und ihre Folgen am sprachlichen Korpus auf diachroner und

\footnotetext{
2 Feministische Ideen, die in den 80er- und 90er-Jahren in Europa in verschiedenen Ländern und durch verschiedene Gremien artikuliert wurden, haben zunächst wenig Resonanz unter polnischen LinguistInnen gefunden. Die Ursache liegt vermutlich zum Teil in der negativen Konnotierung, die dem Begriff von Anfang an im medialen Diskurs in Polen verliehen wurde, zum Teil in der Distanz zum Radikalismus in der Forderung mancher sprachpolitischen Lösungen, die verschiedene feministische Gruppen zu ihren Kampfparolen gemacht haben. Vgl. dazu auch den Titel der ersten linguistischen Konferenz in Polen, die dem Phänomen der Genderproblematik gewidmet wurde, in dem das Adjektiv feministisch durch feminologisch ersetzt wurde (Pierwsza Ogólnopolska Konferencja Feminologiczna, Lublin 1989). So wird auch in manchen späteren Publikationen diese eindeutige Ausweichstrategie angewandt, um den nach wie vor mehr oder weniger unbequemen Begriff zu umgehen oder seine Bedeutung durch Verwendung von Anführungszeichen oder der Formel sogenannt zu entschärfen (cf. Nowosad-Bakalarczyk 2009; Umschlagseite).
}

ISSN 1615-3014 
synchroner Ebene nachvollziehen; die bis jetzt umfangreichste Monographie zum androzentrischen Sprachgebrauch, die von ihnen vorgelegt wurde, möchten die beiden Forscherinnen nur als berichtend und kritisch betrachten und nicht im Forschungsparadigma der feministischen Linguistik platziert sehen. Außerdem sind sie der Ansicht, dass das politische Klima in Polen zum Zeitpunkt der Veröffentlichung ungünstig war, um eine nichtsexistische Sprachreform einzuleiten und durchzuführen sowie damit eine symmetrische Ausdrucksweise zu fordern und zu fördern (cf. Karwatowska/Szpyra-Kozłowska 2005: 275); die Gründe hierfür suchen sie in der Spezifik des polnischen Sprachsystems sowie in der allgemein negativen Wertung zwar geschlechtergerechter, aber noch nicht normgerechter Ausdrücke (cf. Karwatowska/Szpyra-Kozłowska 2005: 281). Auch eine frühere Diskussion während der wissenschaftlichen Konferenz ${ }^{3}$, die 2003 an der Maria-Curie-SkłodowskaUniversität in Lublin veranstaltet wurde und den Problemen der Geschlechterkommunikation gewidmet war, hat gezeigt, dass man in linguistischen Kreisen nur ungern einen unmittelbaren Zusammenhang zwischen sprachlichen Asymmetrien und der Ungleichbehandlung beider Geschlechter in der Gesellschaft sieht. Vielmehr ist man eher geneigt, mit fehlenden oder unzureichenden wissenschaftlichen Beweisen zu argumentieren oder aber auch Argumente aus anderen Wissenschaftsdisziplinen anzuführen, die der feministischen Argumentation entgegengehalten werden (cf. Bartmiński/Majer-Baranowska 2005). Dabei muss aber betont werden, dass das geschlechterspezifische Forschungsparadigma meist aus literatur- und kulturwissenschaftlichem sowie soziologischem und psychologischem Blickwinkel ausgelegt wird; an linguistischen Untersuchungen im feministischen Sinn besteht stets ein großer Nachholbedarf. So ist der Androzentrismus im Polnischen mittlerweile auch Gegenstand einiger deutsch- und englischsprachiger Fachpublikationen geworden, die in Polen und im Ausland veröffentlicht wurden, in denen mit dem Begriffsinstrumentarium der linguistischen Genderforschung und feministischen Methoden gearbeitet wird (cf. Jaworski 1986; Miemietz 1993; 1996; Bochenek 2010; Bulawka 2013).

\section{Feministische Linguistik in der BRD im Kontext neuester sprachlicher Entwicklungen in Polen}

In der feministisch orientierten Linguistik wird die grundlegende Hypothese angenommen, dass "die Sprachen patriarchalisch organisierte Systeme sind, die Entpatrifizierung und Feminisierung bedürfen" (Pusch 1990: 75). Es geht nicht darum, dass Sprachen nur beschrieben werden, wie das die Systemlinguistik macht, sondern dass Sprachen und ihre Anwendung auch im Hinblick auf das Vorhandensein und somit die kommunikative Repräsentanz des weiblichen Elements kritisch bewertet werden. In diesem Zusammenhang werden vor allem solche Situationen fokussiert, in denen Frauen nicht die gleichen Chancen des Gemeintseins wie Männer haben, d.h. (a) auf Frauen und Männer unterschiedlich referiert wird, die Verwendung vom generischen Maskulinum Frauen und ihre Leistungen in der jeweiligen Sprache unsichtbar macht, (b) maskuline und feminine Ausdrücke asymmetrisch sind und (c) die Bezeichnung einer Frau mit einer maskulinen Form als Aufwertung sowie die Bezeichnung eines Mannes mit einer femininen Form als Degradierung interpretiert wird (cf. Hellinger 1985: 3-4). Diese Charakteristika sind nicht nur der deutschen

3 Gemeint ist das V Forum Kultury Słowa: Polak z Polakiem. Porozumiewanie się. Bariery i pomosty, das vom 16.-18.10.2003 in Lublin stattgefunden hat. 
Sprache eigen, sondern verschiedenen Sprachen der Welt, unabhängig von ihrer Herkunft, Entwicklung und typologischen Struktur. Auch im Polnischen findet man Beispiele, die in dieser Hinsicht besondere Parallelitäten zu anderen Sprachen aufweisen:

a) (dt.) Wer ist der Autor dieser Abhandlung? / (poln.) Kto jest autorem tej rozprawy?

b) (dt.) Sekretär - Sekretärin / (poln.) sekretarz - sekretarka

c) (dt.) Er heult wie ein Weib. / (poln.) Ryczy jak baba.

Die genderorientierte Kritik ist eine Sprachkritik, die sich einerseits auf verschiedene sprachliche, d. h. morphologische, syntaktische und lexikalische Einzelerscheinungen sowie Inkonsequenzen und Lücken in einzelnen Subsystemen der Sprache bezieht, andererseits ihre Aufmerksamkeit auf Einzelsprachen als komplexe Gebilde lenkt und "aus der Beschreibung sprachlicher Asymmetrien die Forderung nach dem Abbau der sprachlichen und gesellschaftlichen Diskriminierung von Frauen ableitet" (Hellinger 1990: 12). Das betrifft im gleichen Umfang das Deutsche wie das Polnische (cf. Nowosad-Bakalarczyk 2003: 250-253). Im Mittelpunkt des Interesses der genderorientierten Sprachkritik steht die sexistische Sprache, wenn sie "Frauen und ihre Leistungen ignoriert, wenn sie Frauen nur in Abhängigkeit von und Unterordnung zu Männern beschreibt, wenn sie die Frauen nur in stereotypen Rollen zeigt und ihnen so über das Stereotyp hinausgehende Interessen und Fähigkeiten abspricht, wenn sie die Frauen durch herablassende Sprache demütigt und lächerlich macht" (Frank-Cyrus 1998: 70). Lexikalisch-semantische Analysen haben z. B. ergeben, dass die formal-logische Struktur der maskulinen und femininen Personenbezeichnungen historisch tief in der sozialen Hierarchie verankert ist und sich als eine Form der sprachlichen Gewalt ${ }^{4}$ manifestiert (cf. Samel 2000: 129). Kritisiert wird vor allem der Gebrauch des generischen Maskulinums (das generische Maskulinum wird verwendet, um beide Geschlechter zu nennen, z. B. (dt.) die Lehrer, die Autoren - (poln.) nauczyciele, autorzy); die deutsche und die polnische Grammatik ${ }^{5}$ schreiben vor, dass "jede noch so große Menge von Frauen symbolisch zu einer Männermenge wird, sobald ein einziger Mann hinzukommt. So werden aus 99 Sängerinnen und einem Sänger auf Deutsch (und auf Polnisch - M. C.) einhundert Sänger" (Pusch 1998: 12); das generische Maskulinum wird abgelehnt, weil maskuline Formen, die generisch verwendet werden, an Männer denken lassen und Frauen samt ihren Leistungen unsichtbar machen. Der Kritik wird auch die

4 Samel (2000, 130-131) interpretiert in Anlehnung an Homberger (1993) und Trömel-Plötz (1984) die sprachliche Gewalt, die von Männern Frauen gegenüber ausgeübt wird, als personale (wenn Männer Frauen nicht ausreden lassen oder selbst die Gesprächsthemen bestimmen), psychische (wenn Frauen im öffentlichen Diskurs der Männer sprachlich definiert und fixiert werden), subtile (wenn das männliche Sprachverhalten auf typische Verhaltensweisen zurückzuführen ist, die aus der kulturellen Entwicklung einer Gesellschaft resultieren, aber gesellschaftlich akzeptiert werden) und strukturelle Gewalt (die mit strukturellen Eigenschaften einer jeweiligen Sprache zusammenhängt).

5 In aktuellen Publikationen wird mit Rücksicht darauf das Verhältnis von Genus und Sexus thematisiert, worauf in diesem Beitrag nicht näher eingegangen wird. Mein Interesse gilt weniger systemspezifischen Fakten (z. B. dem System der Genera oder der Formenbildung in den beiden Sprachen ), die in kontrastiven Grammatiken explizit beschrieben wurden und nur wenig Explanationspotential für die vorliegende Untersuchung liefern können, sondern sich verändernden Gebrauchsweisen, in denen bis jetzt gültige Sprachnormen bewusst verletzt werden. Solche Normverletzungen betrachte ich als Ergebnisse zielgerichteter sozialer Handlungen, die diese Normen auf Grund neuer Entwicklungen in Frage stellen und alternative Lösungen verlangen. Es bleibt die Aufgabe der Öffentlichkeit, sich mit diesem soziolinguistischen Phänomen auseinanderzusetzen.

ISSN 1615-3014 
Movierung mittels -in unterzogen, denn alle movierten Feminina ${ }^{6}$ mit -in avisieren - wie die feministische Sprachkritik ${ }^{7}$ mehrfach betont hat - die sprachliche Abhängigkeit der Frau von dem Mann, auch wenn aus gegenwartssprachlicher Sicht gegen das Suffix -in eigentlich nichts mehr einzuwenden ist, weil es inzwischen die Bedeutungskomponente 'Zugehörigkeit zu etwas' verloren hat und neutral zu werten ist (cf. Werner 2007: 8). Die Möglichkeit, movierte Feminina im Polnischen zu bilden, ist zwar gegeben ${ }^{8}$, aber sie ist oft - semantisch gesehen - mit restriktiv wirkenden Regeln verbunden (neben stolarz gibt es z. B. auch stolarka. Stolarz bezeichnet 'Schreiner' und 'Schreinerin' zugleich; stolarka hat in der Allgemeinsprache die Bedeutung 'Schreinerei'), was in nicht seltenen Fällen zu negativ konnotierten, ungewöhnlich wirkenden Bildungen führen kann. Im Polnischen gibt es viele feminine Suffixe (cf. Engel 2000: 731-733) und "keines von ihnen spielt auch nur annähernd eine so dominierende Rolle wie das deutsche Suffix -in" (Engel 2000: 734). U. a. daraus können sich manche Schwierigkeiten in der Distribution der gängigen Suffixe ergeben. Die kommunikative Sprachpraxis von heute ${ }^{9}$ zeigt jedoch, dass neue Gebrauchspräferenzen allmählich die erstarrten sprachlich-kulturellen Normen zu unterspülen beginnen und deren Wandel zur Folge haben. So müsste auch die Diagnose der polnischen LinguistInnenGermanistInnen, die um die Wende des 20. und 21. Jh. formuliert wurde und in Opposition zu Ansichten der polnischen LinguistInnen-PolonistInnen aus Język Polski und Poradnik Językowy steht, revidiert werden, nach der die movierten Formen psycholożka (Psychologin) und filolożka (Philologin) im Polnischen als unkorrekt bezeichnet werden (cf. Engel 2000: 735).

Sprachen sind keinesfalls neutrale Kommunikationsmittel, sondern spiegeln immer hierarchische Machtstrukturen und soziale Ungleichheiten wider; sie beeinflussen und formen unser Denken und die Wahrnehmung, sie können kontrastive Vorstellungen erzeugen und alternative Realitäten schaffen (cf. Frank-Cyrus 1998: 71). Mit der Kritik an sexistischen Spracherscheinungen im Sprachsystem und Sprachgebrauch haben FeministInnen in Deutschland einen Sprachwandel eingeleitet, der weit über den Bereich der Berufs- und Personenbezeichnungen hinausgeht und im gleichen Maße nahezu alle Bereiche des gesellschaftlichen Lebens erfasst hat (offizielle Sphäre) sowie die Mentalität des Durchschnittsbürgers (persönliche Sphäre) (cf. Samel 2000: 88). In allen Bereichen des öffentlichen Lebens ist mittlerweile die Doppelnennung üblich, die den Kommunizierenden erlaubt, beide Geschlechter gleichermaßen anzusprechen. Noch Anfang der 90er-Jahre hat die Arbeitsgruppe Rechtssprache drei Kritikpunkte vorgebracht, an denen der Deutsche

\footnotetext{
6 Die linguistische Diskussion, die die Bildung femininer Berufsbezeichnungen betrifft, hat im Deutschen eine lange Tradition. Näheres dazu bereits bei Samel (2000: 101-104).

7 In der früheren Diskussion in Deutschland wurden dazu auch radikale Meinungen formuliert. Bei Pusch (1984: 54) wurde das deutsche Sprachsystem „eine eklatante Fehlkonstruktion“ im Hinblick auf die Movierung auf -in genannt, die „absurd und unökonomisch“ ist, weil sie das männliche als Norm und das weibliche als von der Norm abgeleitet vorsieht. Pusch schlug die Abschaffung des Suffixes -in sowie Einführung des generischen Femininums und der Neutralisation vor (vgl. Pusch 1984: 59); wegen der darauffolgenden Kritik wurde der kontroverse Vorschlag zurückgezogen.

8 Das Polnische verfügt über zwei produktive Wortbildungssuffixe für die Bildung movierter Feminina: $-a$ (Endung) und $-k a$ (Formans).

$9 \mathrm{Zu}$ neuesten Tendenzen in der Bildung und Verwendung von femininen Formen im Polnischen vgl. bei Nowosad-Bakalarczyk (2009: 143-157).
} 
Frauenrat ${ }^{10}$ festhielt und sich mit seinen Ideen weitgehend durchgesetzt hat. Auf die Verwendung des generischen Maskulinums in der Amtssprache wird so weit wie möglich verzichtet. Die früher übliche Anrede unverheirateter Frauen mit Fräulein wurde abgelöst (cf. Klann-Delius 2005: 187-188). All diese Veränderungen haben besonders den öffentlichen Sprachgebrauch erfasst, z. B. Stellenanzeigen, Gesetzestexte, Bekanntmachungen, Merkblätter und Formulare. Veränderungen in der Gesetzgebung haben u. a. zur Geschlechtsspezifizierung bzw. Neutralisierung bei den Berufsbezeichnungen sowie Amtsund Dienstbezeichnungen (z. B. Minister, Ministerin) beigetragen; im Vordergrund stehen immer die sprachliche Sichtbarmachung von Frauen und die Gleichbehandlung beider Geschlechter (cf. Hellinger 1993: 5). Bereits 1979 wurde bundesweit rechtlich geregelt, dass künftig maskuline und feminine Berufsbezeichnungen in deutschen Ausbildungsordnungen verwendet werden müssen, ein Jahr später wurde das Bürgerliche Gesetzbuch um den Paragrafen $611 \mathrm{~b}$ ergänzt, nach dem "Arbeitsplätze zunächst nicht mehr allein für Männer oder allein für Frauen ausgeschrieben werden sollten". Nach und nach wurden auch verschiedene Empfehlungen und Richtlinien ${ }^{11}$, Broschüren, Gesetze und journalistische Glossen herausgegeben, u. a. Richtlinien zur Vermeidung sexistischen Sprachgebrauchs, Leitfaden zur geschlechtergerechten Formulierung, der Bericht des Deutschen Bundestages über Maskuline und feminine Personenbezeichnungen in der Rechtssprache, Sprachliche Gleichbehandlung von Frauen und Männern, Eine Sprache für beide Geschlechter - Richtlinien für einen nichtsexistischen Sprachgebrauch. Die Empfehlungen und Richtlinien ${ }^{12}$ dokumentieren eigentlich nicht nur, wie geschlechtergerechtes Formulieren zu tätigen ist, sondern ermuntern auch zu einem größeren Sprachbewusstsein (cf. Braun 2000: 4). Ihr Ziel ist es vor allem, "sexistische Sprache zu identifizieren und alternative Gebrauchsweisen anzubieten, die nicht frauenfeindlich und diskriminierend sind" (Klann-Delius 2005: 184). Außerdem enthalten sie wertvolle Hinweise, Ratschläge und Musterbeispiele, wie mit verschiedenen Formen der Gleichbehandlung sprachlich umgegangen werden kann (cf. Samel 2000: 141).

\section{Deutsche und polnische Stellenausschreibungen im Kontrast}

Im Folgenden werde ich auf das Problem der sprachlichen Gleichbehandlung von Frauen und Männern in deutschen und polnischen Stellenausschreibungen eingehen.

10 Diese drei Kritikpunkte betreffen: die Diskriminierung der Frauen durch den Gebrauch von maskulinen Berufs-, Amts- und Funktionsbezeichnungen; die Tatsache, dass die Rechtssprache tradierte Rollen der beiden Geschlechter verfestigt; den negativen Einfluss der Rechtssprache auf die gesellschaftliche Arbeits- und Rollenverteilung und damit einhergehend die Erschwerung gesellschaftlicher Veränderungen (vgl. Samel 2000: 112).

11 Erste Richtlinien zur Vermeidung sexistischen Sprachgebrauchs wurden in Deutschland im Jahre 1980 von Ingrid Guentherodt, Marlis Hellinger, Luise Pusch und Senta Trömel-Plötz formuliert. Später haben auch verschiedene Institutionen und Organisationen eigene Regelungen erarbeitet. Die Duden-Redaktion hat über die Möglichkeiten und Probleme geschlechtergerechten Formulierens zum ersten Mal in der Duden-Ausgabe 1997 referiert (vgl. Feigl 2009: 32).

12 Das Bundesverwaltungsamt hat im Jahre 2002 das BBB-Merkblatt M 19 Sprachliche Gleichbehandlung von Frauen und Männern. Hinweise, Anwendungsmöglichkeiten und Beispiele herausgegeben, in dem Empfehlungsschriften und Erlasse zur sprachlichen Gleichbehandlung in Bund, Ländern und Kommunen verzeichnet werden.

ISSN $1615-3014$ 
Seitdem in Deutschland das Allgemeine Gleichbehandlungsgesetz in Kraft getreten ist, ist jede Stellenausschreibung geschlechtergerecht zu formulieren, d. h. die Formulierung muss so gestaltet sein, dass sie beide Geschlechter in gleichem Maße anspricht und weder das eine noch das andere Geschlecht (von nur wenigen Ausnahmen abgesehen) bevorzugt wird. In Polen gibt es solche Regelungen ${ }^{13}$ noch nicht. In die Debatte über die Verwendung neuer femininer Formen hat sich zwar in letzter Zeit auch der polnische Sprachrat eingeschaltet, aber seine Interessen betreffen formal-semantische Unsicherheiten bzw. kulturgeprägte Hindernisse, mit denen man beim Gebrauch neuer femininer Derivate konfrontiert wird (maskuline Berufsbezeichnungen werden z. B. als prestigereicher und statushöher, feminine Bildungen dagegen als herabwürdigend, kampffeministisch und künstlich angesehen); weder sprachpolitische Entscheidungen werden getroffen noch praktische Hinweise für die Allgemeinnutzung femininer Formen im offiziellen Sprachgebrauch unterbreitet, die den Vorschlägen polnischer feministischer LinguistInnen entgegenkommen würden ${ }^{14}$.

Um jedoch einen Einblick in das Problem zu geben ${ }^{15}$, habe ich eine Korpusdatei erstellt, die aus 200 Stellenausschreibungen besteht (jeweils 100 in deutscher und polnischer Sprache), die (a) in der Online-Ausgabe der deutschen überregionalen Tageszeitung "Süddeutsche Zeitung" im März 2011 und (b) im Internetportal "Pracuj" (dt. "Arbeite!") im April 2013 publiziert wurden ${ }^{16}$. Beide Teildateien sind - quantitativ gesehen - gleich. Sie enthalten jeweils Stellenausschreibungen, die in Digitalform erstellt wurden und unterschiedlichen Umfangs sind. Die Gesamtdatei wurde nach dem Prinzip der Zufallsauswahl zusammengestellt, d. h. jede Stellenbeschreibung hatte die gleiche Wahrscheinlichkeit, in die Stichprobe zu gelangen, und die Ziehungen erfolgten unabhängig voneinander. Bei der durchzuführenden Analyse werden die beiden Teildateien aufeinander bezogen, um bestehende Kontraste zwischen deutschen und polnischen Stellenausschreibungen unter Bezugnahme auf geschlechtergerechtes Formulieren $\mathrm{zu}$ erfassen und $\mathrm{zu}$ beschreiben; im Weiteren soll auch überprüft werden, ob, und wenn ja, inwiefern neu entstandene, feminine Wortbildungen Eingang in die offizielle Sphäre der Kommunikation gefunden haben.

Eine Stellenausschreibung (= StA) ist eine weitgehend konventionalisierte Beschreibung einer $\mathrm{zu}$ besetzenden Arbeitsstelle; sie gibt Auskunft über das gewünschte Anforderungsprofil sowie eine erforderliche Ausbildung, Fähigkeiten, Eigenschaften und weitere

\footnotetext{
13 In der polnischen Gleichstellungspolitik wird die geschlechtergerechte Sprache nicht berücksichtigt. Ich meine damit rechtliche Regelungen, die neueste Entwicklungen im polnischen Sprachgebrauch, denen man sich heute nur schwer widersetzen kann, sanktionieren können. Vgl. dazu einen Beitrag von Chołuj/Neusüß (2004: 182-193), in dem der Stand der Gleichstellungspolitik für das Jahr 2004 dokumentiert wird.

14 Eine kritische Stellung zur Argumentation des polnischen Sprachrates ist bei Belczyk-Kohl (2013: 30-38) zu finden.

15 Untersuchungen zu Stellenausschreibungen hat bereits Miemietz (1993) vorgenommen. In diesem Kontext sind auch Forschungsanalysen von Nowosad-Bakalarczyk zu sehen, die 2001 aus der Gazeta Wyborcza exzerpierte Stellenausschreibungen im Hinblick auf den Adressatenkreis erforscht und fragt, welche von ihnen an Frauen (1. Gruppe), welche an Männer (2. Gruppe) und welche an Frauen und/oder Männer (3. Gruppe) adressiert sind, ohne auf das Problem der geschlechtergerechten Ausdrucksweise einzugehen (vgl. NowosadBakalarczyk 2009: 72-87).

16 Die deutschsprachige Datei habe ich der Magisterarbeit von Anna Maciejewska entnommen (cf. Literaturverzeichnis), die 2012 im Rahmen des von mir am Institut für Germanistik der Universität in Bydgoszcz (Polen) geführten Seminars Sprache und Geschlecht entstanden ist und erfolgreich verteidigt wurde.
} 
Voraussetzungen; sie ist ein spezifischer Sprechakt, dem eine bestimmte Intention zugrunde liegt (persuasive Funktion), die durch den Vollzug des Sprechaktes ausgedrückt wird. Die StAen lassen sich den folgenden drei Kategorien zuordnen, mit denen in der feministischen Fachliteratur (vgl. Püsch 1990; Samel 2000) über Probleme des gendergerechten Sprachgebrauchs referiert wird:

1) geschlechtergerechte StAen

Nach Feigl (2009: 31) sind geschlechtergerechte StAen durch folgende Kriterien gekennzeichnet:

- Vermeidung des generischen Maskulinums;

- $\quad$ sprachliche Sichtbarmachung des Geschlechts - überall, wo Frauen beteiligt sind, soll dies auch sprachlich ausgedrückt werden;

- sprachliche Symmetrie - auf Frauen und auf Männer soll gleichermaßen Bezug genommen werden;

- Verwendung geschlechtsneutraler Begriffe.

2) geschlechtsspezifische StAen

Geschlechtsspezifische StAen sind entweder an Frauen oder Männer adressiert. Feigl (2009: 7) macht in diesem Zusammenhang u. a. darauf aufmerksam, dass sich geschlechtsspezifische StAen für ein Unternehmen als nachteilig erweisen können, denn dabei wird indirekt suggeriert, dass das Kriterium Geschlecht für eine bestimmte Position wichtiger als ihre Eignung ist. Außerdem muss man auch damit rechnen, dass die Zahl der potentiellen Bewerbungen geringer sein kann und damit die Chance, eine geeignete Person für die ausgeschriebene Stelle zu finden.

3) inkonsequente (diskriminierende) StAen

Für diese StAen ist charakteristisch, dass sie sprachlich inkonsequent formuliert sind, sodass a) entweder Frauen angesprochen und Männer diskriminiert oder b) Männer angesprochen und Frauen diskriminiert werden.

Im deutschen Teilkorpus lassen sich alle 3 Typen von StAen nachweisen (cf. Maciejewska 2012: 32-57):

- geschlechtergerechte (71\%),

- geschlechtsspezifische (14\%) und

- inkonsequente (diskriminierende) StAen (15\%).

Im polnischen Teilkorpus gibt es keine geschlechtergerechten StAen. Repräsentiert werden lediglich geschlechtsspezifische (68\%) und inkonsequente (diskriminierende) StAen (32\%).

\subsection{Geschlechtergerechte Stellenausschreibungen}

Insgesamt wurden 71 geschlechtergerechte StAen ermittelt. Im Deutschen gibt es bekanntlich mehrere formal-sprachliche Möglichkeiten, die auch sprachpraktisch genutzt werden, um Frauen und Männer in StAen gleichermaßen anzusprechen. 
a) das Hinzufügen von "m/w" (52\%)

Hierfür wurden 37 StAen exzerpiert, in denen beide Geschlechter durch das Hinzufügen von "m/w" (männlich/weiblich) geschlechtergerecht behandelt werden; diese Möglichkeit wird oft genutzt und hat sich als die produktivste erwiesen. In der überwiegenden Zahl der StAen, die sich dieser Kategorie zuordnen lassen, wird die maskuline Form (das generische Maskulinum) (z. B. Auftragssachbearbeiter) mit dem Zusatz "m/w" (auch "w/m") verwendet.

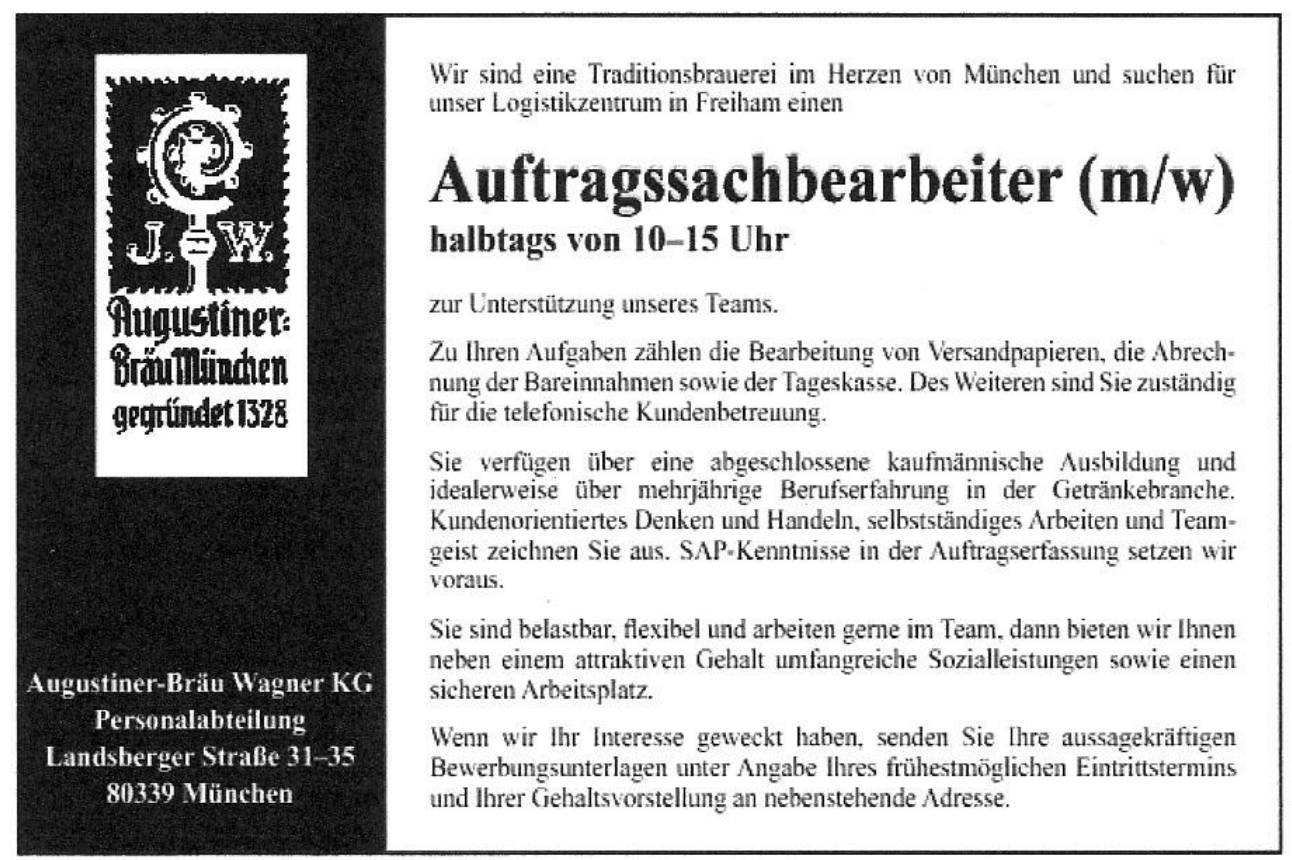

stellenmarkt.sueddeutsche.de, 05.03 .2011

\section{Abbildung 1}

b) der Schrägstrich $(30 \%)$

Im deutschen Teilkorpus sind insgesamt 21 Belege verzeichnet, in denen der Schrägstrich in geschlechtergerechten StAen (z. B. Versorgungsingenieur/in) genutzt wird. Eine wichtige Rolle spielt dabei der sprachökonomische Aspekt. 


\section{0}

\section{Böblingen bietet Arbeit}

Die Stadtwerke Böblingen versorgen als Eigenbetrieb der großen Kreisstadt Böblingen rund 46.500 Einwohner mit Wasser und Fernwärme. Darüber hinaus wird die Abwasserbeseitigung, Bäder, Parkhäuser und Park \& Ride-Anlagen sowie ein Industriegleis betrieben. Für den Bereich Wasser- und Fernwärmeversorgung suchen wir zumn nächstmöglichen Zeitpunkt eine/n

\section{Versorgungsingenieur/in.}

Zu lhren Aufgabengebieten der Fernwärme und Wasserversorgung gehören u. a.

- Ausarbeitung und Bewertung von umfangreichen und komplexen Planungskonzepten

- Planung von Neuinvestitionen

- Optimierung der bestehenden Versorgungsanlagen und deren Betrieb

- Projektbearbeitung in allen Leistungsphasen

Unsere Anforderungen

- Abgeschlossenes Studium der Versorgungs-, Gebäude- oder Energietechnik

- Mindestens 5-jährige Berufserfahrung

- Erfahrung im Projektmanagement

- Selbstständige und strukturierte Arbeitsweise

- Hohe Einsatzbereitschaft und Belastbarkeit

- Bereitschaft zur aktiven Mitarbeit bei der Optimierung betrieblicher Abläufe

- Teamfähigkeit

Wir bieten ein vielseitiges und interessantes Aufgabengebiet, das selbstständiges und eigenverantwortliches Arbeiten im Team ermöglicht. Die leistungsgerechte Vergütung erfolgt je nach Qualifikation und persönlicher Voraussetzung nach dem TVöD.

Wir freven uns auf thre aussagefähige Bewerbung bis spätestens 15. April 2011 an die Stadtverwaltung Böblingen, Haupt- und Presseamt, Personalabteilung, Posttach 1920, 71009 Böblingen. Für weitere Intormationen steht thnen Frau Kaebert, Tel. 07031/2192-64 von den Stadtwerken Böblingen, gern zur Verfügung.

stellenmarkt.sueddeutsche.de, 13.03.2011

\section{Abbildung 2}

c) neutrale Formen ( $9 \%)$

Registriert wurden 6 verschiedene StAen mit neutralen Formen, die durch das Hinzufügen von -kraft (z. B. Lehrkraft) und -hilfe (z. B. Bürohilfe) gebildet wurden. Neutrale Formen machen zwar das Geschlecht unsichtbar, kommen aber dem Prinzip der sprachlichen Gleichbehandlung beider Geschlechter entgegen. 


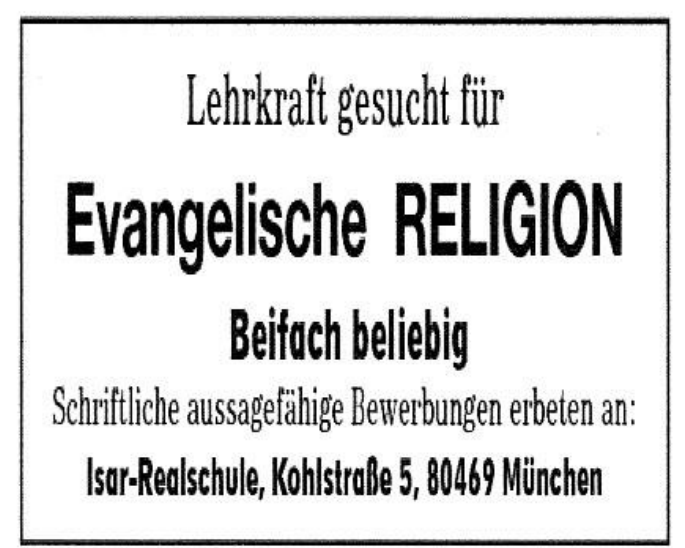

stellenmarkt.sueddeutsche.de, 03.03 .2011

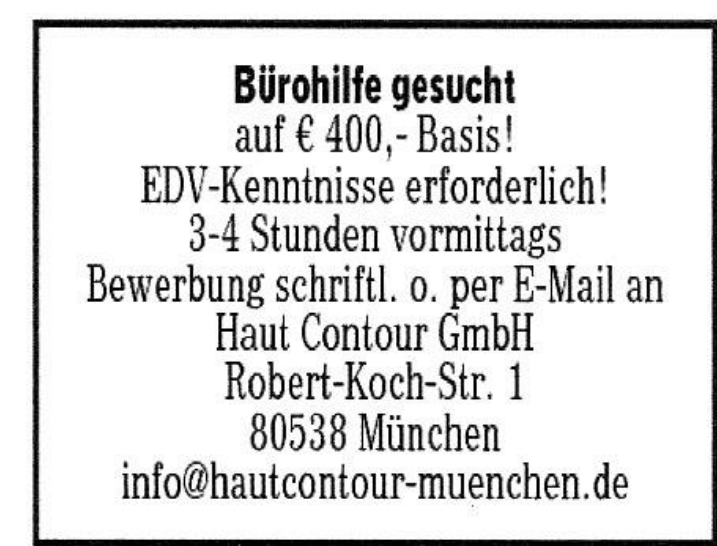

stellenmarkt.sueddeutsche.de, 10.03 .2011

\section{Abbildung 3}

d) das Binnen-I (4\%)

Im Korpus wird in 3 StAen das Binnen-I zur geschlechtergerechten Abbildung eingesetzt; funktionell gesehen kommt das Binnen-I dem Streben nach Sprachökonomie entgegen und wird vor allem, was sich anhand des Korpus nachweisen lässt, in Kleinanzeigen verschiedener Art verwendet ${ }^{17}$.

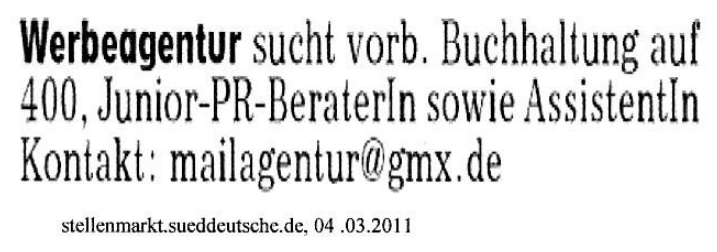

Abbildung 4

e) die Beidnennung (4\%)

Das Korpus enthält 3 StAen, in denen die Beidnennung (z. B. eine Beamtin/einen Beamten) enthalten ist. In der Hierarchie der Möglichkeiten, beide Geschlechter sprachlich gleich zu behandeln, steht die Beidnennung zusammen mit dem Binnen-I erst an vorletzter Stelle. 


\section{GEMEINDE GRÖBENZELL}

Die Gemeinde Gröbenzell, ca. 19.500 Einwohner, am westlichen Stadtrand von München an der S-Bahnlinie 3 gelegen, sucht zum schnellstmöglichen Zeitpunkt

\section{eine Beamtin/einen Beamten}

in der 3. Qualifikationsebene (ehemals gehobener nichttechnischer Verwaltungsdienst) bzw. Verwaltungsfachangestellte/r AL II

in Vollzeit für die gemeindliche Bauverwaltung.

\section{Ihre Aufgaben:}

- Beratung der Bürger im Bauvollzug

- Erstellung Genehmigungsfreistellungsbescheide

- Überprüfung Bauanträge, Vorbescheide und Freistellungsanträge auf Vollständigkeit und Genehmigungsfähigkeit

- Erstellung der Beschlussvorlagen für den Bauausschuss

- Vollzug der gemeindlichen Gestaltungssatzungen hinsichtlich Überwachung und Bußgeldverfahren

- Erstellung Bescheide von isolierten Befreiungen von Bebauungsplänen

- Mitwirkung in der Bauleitplanung (Bebauungspläne)

- Vertretung des Sachgebietsleiters der Bauverwaltung

- Sizungsdienst generell Bauausschuss und bei Vertretung des Sachgebietsleiters auch Planungsausschuss und Gemeinderat

Was wir von thnen erwarten:

- fundierte EDV-Kenntnisse (Word, Excel, Outlook, GIS)

- Erfahrung im Bereich der Bauverwaltung (vor allem im gesamten Bauvollzug)

- Organisations- und Planungsgeschick

- sicheres und höfliches Auttreten im Rahmen des Parteiverkehrs

- Teamfähigkeit

- Bereitschaft für Dienst zu ungünstigen Zeiten (Sitzungsdienst)

Schwerbehinderte werden bei gleicher Eignung und Befähigung vorrangig berücksichtigt.

Die Gemeinde Gröbenzell möchte in diesem Bereich den Frauenanteil gerne erhöhen und ist deshalb besonders an Bewerbungen von Frauen interessiert.

Ihre aussagefähige Bewerbung mit Zeugniskopien und Lebenslauf senden Sie bitte bis spätestens 26. April 2011 an die Gemeinde Gröbenzell, Rathausstraße 4, 82194 Gröbenzell. Telefonische Auskünfte erteilen Frau Pfeffer-Walk, Telefonnummer 081 42/5 $05-47$ und Herr Pfannes, Telefonnummer 081 42/5 05-48.

stellenmarkt.sueddeutsche.de, 09.03.2011

\section{Abbildung 5}

f) das Splitting mittels Klammern (1\%)

Etablierte, dynamische Kanzlei mit interessanter Klientel (auch international) sucht baldmöglichst eine( $n$ )

Steuerberater(in) / Steuerberateranwärter(in) qualifizierte(n) Steuergehilfen(in)

mit Berufserfahrung zur selbständigen Betreuung eines abgegrenzten, anspruchsvollen Mandantenkreises.

Das Aufgabenfeld umfasst $u$. a. die Erstellung von Steuererklärungen und Jahresabschlüssen sowie laufende Beratungstätigkeiten.

Geipel \& Kollmannsberger Partnerschaft

Wirtschaftsprüfer-Steuerberater-Rechtsbeistand

Geiselgasteigstr. 124, 81545 München

Kontakt: Stefanie Schroedter, o 089/642444-19

Email: s.schroedter@geipel-kollmannsberger.de

stellenmarkt.sueddeutsche.de, 11.03.2011

Abbildung 6

ISSN 1615-3014 
Im Teilkorpus wurde lediglich 1 StA gefunden, in der das Suffix -in in Klammern steht [z. B. Steuerberater(in)/Steuerberateranwärter(in)]. Diese Möglichkeit wird am seltensten genutzt, in manchen Publikationen zur feministischen Sprachkritik wird sie sogar abgelehnt und als zweitrangiges Anhängsel interpretiert (cf. Braun 2000: 10).

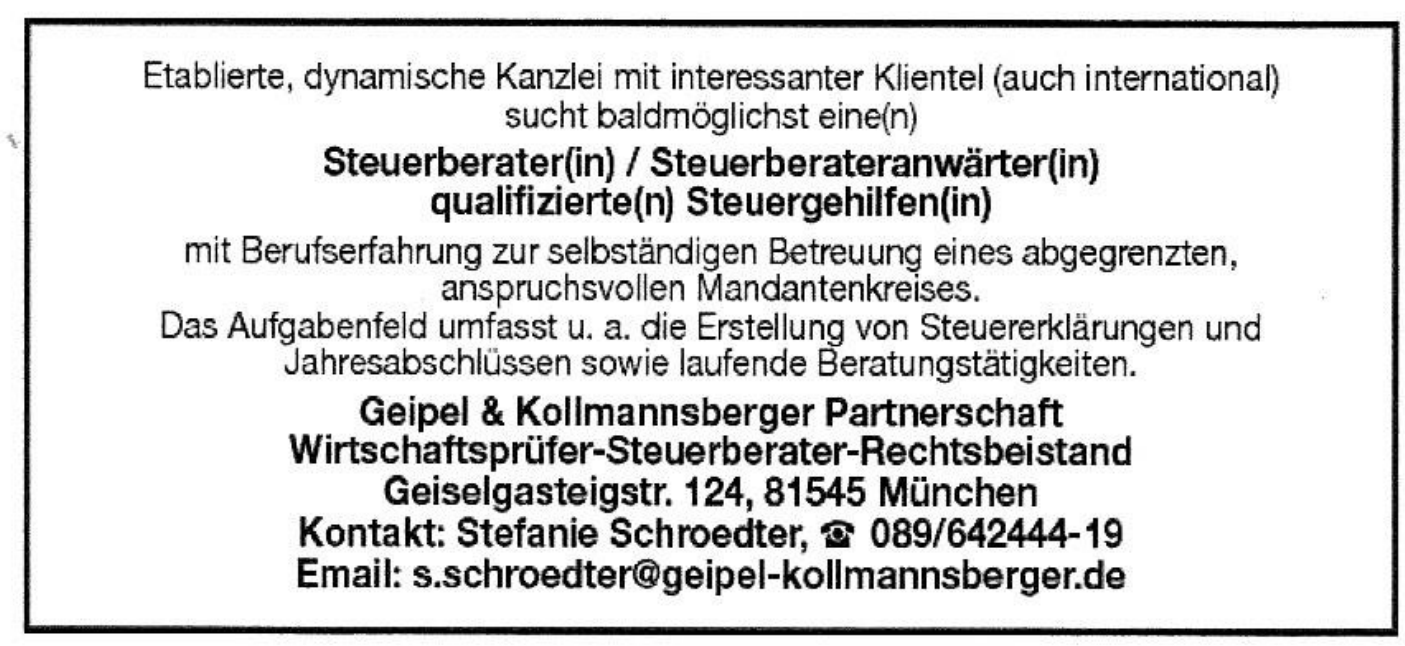

stellenmarkt.sueddeutsche.de, 11.03.2011

\section{Abbildung 7}

\subsection{Geschlechtsspezifische Stellenausschreibungen}

Geschlechtsspezifische StAen aus dem deutschen Teilkorpus sind an beide Geschlechter gerichtet - an Frauen und an Männer. In 14 StAen wird das gewünschte Geschlecht gleich am Anfang signalisiert, was mit der Spezifik der Arbeitsstelle (z. B. Die Aufgabe erfordert die Besetzung der Stellen durch ausschließlich weibliche Fachkräfte/Verkäufer im Außendienst) zusammenhängt. Auf keinen Fall kann hier von der Diskriminierung von Frauen oder Männern gesprochen werden. 


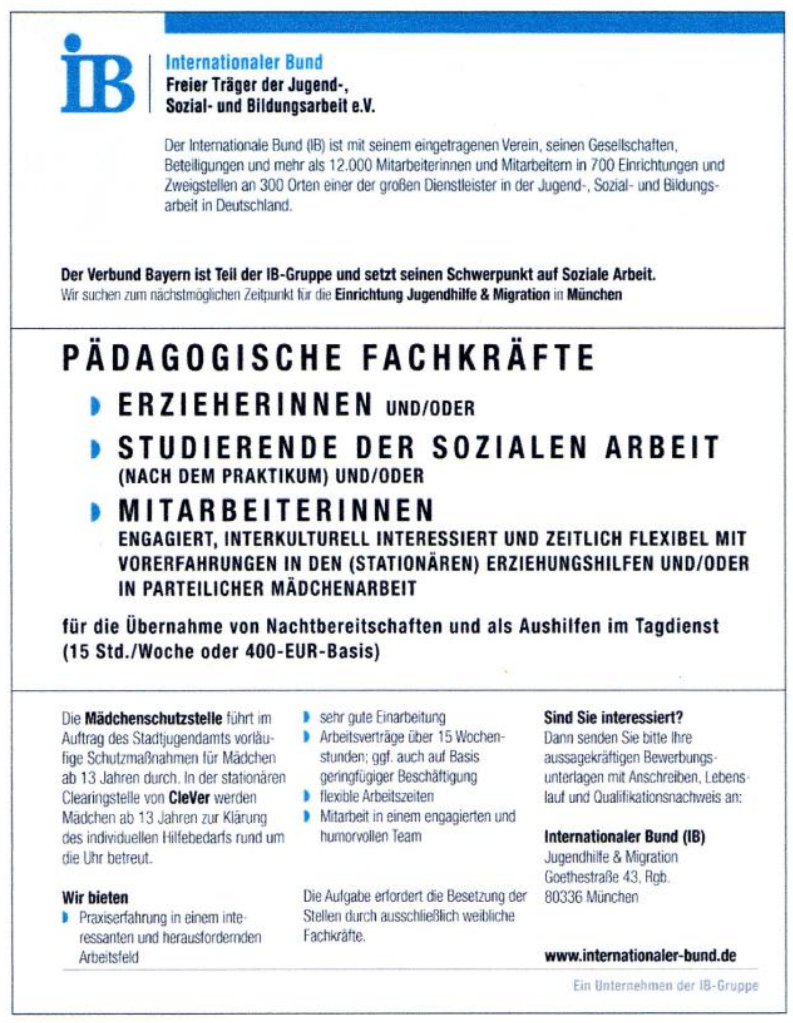

\section{Verkäufer im Außendienst}

Weltweit vertreibt die Firma Present Time GmbH 8 Marken im Bereich Geschenkartikel, Wohnaccessoires und Trendartikel.

Für unser junges Team in Ludwigsburg suchen wir zur sofortigen Verstärkung einen fest angestellten Verkäufer im Außendienst für das Postleitzahlengebiet 8 \& 9 .

Sie sind eine Verkäuferpersönlichkeit mit Spaß am selbstorganisierten Arbeiten, sind belastbar und teamfähig. Des Weiteren besitzen Sie eine abgeschlossene kfm. Ausbildung, mehrere Jahre Berufserfahrung im Außendienst, gute Englischkenntnisse und sind sicher im Umgang mit MS Office!? Dann sind Sie bei uns genau richtig!

Ihre Aufgaben: Kundenbesuche, Neukundenakquise, Präsentation der Marken und Konzepte, Auftragserfassung. Verkauf auf Messen. Wir bieten: Firmenwagen sowie alle nötigen Arbeitsutensilien.

Bitte senden sie thre vollständigen Bewerbungsunterlagen an: Leon Urselmann lu@presenttime.com

Weitere Informationen: www.presenttime.com

stellenmarkt.sueddeutsche.de, 12.03. 2011

Abbildung 8

Im polnischen Teilkorpus gibt es dagegen 68 geschlechtsspezifische StAen, in denen man sich - formal gesehen - nur an Männer wendet ${ }^{18}$. Dabei sind zwei Subklassen zu unterscheiden: (a) in der ersten Teilmenge sind StAen enthalten, aus denen es nicht klar hervorgeht, ob neben Männern auch Frauen mitgemeint sind oder nicht; maskuline Personenbezeichnungen (z. B. technolog drewna) lassen in diesem Fall zwei mögliche Interpretationen $\mathrm{zu}, \mathrm{d}$. h. die generische Interpretation, bei der Frauen sowie Männer mitgemeint sind, und die zweite Interpretation, bei der nur Männer angesprochen werden; (b) in der zweiten Teilmenge gibt es StAen, in denen man sich direkt an Männer (z. B. specjalista ds. wsparcia klienta) wendet. Das ist nicht nur an der Personenbezeichnung zu erkennen, sondern auch an verbalen Formen (z. B. czut się, mógt, wykazywat), die im Polnischen geschlechtsspezifisch sind. Stellenweise werden aber auch neutrale Formen (z. B. osoby) verwendet. Der Unterschied zwischen neutralen Formen im deutschen und polnischen Teilkorpus besteht darin, dass die Neutralisierung im Deutschen durch Neubildungen erfolgt, während im Polnischen sprachübliche neutrale Formen verwendet werden, an denen sich das Geschlecht nicht identifizieren lässt. Diese Bemerkung gilt in gleichem Maße auch für andere neutrale Bezeichnungen, die in inkonsequenten StAen enthalten sind.

18 In Deutschland wurden Untersuchungen durchgeführt, denen zu entnehmen ist, dass StBen, die im generischen Maskulinum formuliert sind, Frauen davon abhalten, sich um eine Arbeitsstelle zu bewerben (vgl. Stalberg/Sczesny 2001: 139). 


\section{SKLEJKA \\ MULTI SA
Bydgoskie Zakłady Sklejek "Sklejka-Multi" S.A. ul. Fordońska 154, 85-752 Bydgoszcz
poszukuje pracownika na stanowisko:

\section{TECHNOLOG DREWNA}

Oferujemy:

- ciekawe, dynamiczne i interesujące wyzwania zawodowe,

- stabilność zatrudnienia

- możliwość rozwoju zawodowego,

- profesjonalne szkolenia

Wymagamy:

- wyższego wykształcenia kierunkowego (Mechaniczna Technologia Drewna),

- doświadczenia zawodowego,

- bardzo dobrej znajomości języka angielskiego ( znajomość j. niemieckiego będzie

dodatkowym atutem),

- dobrej organizacji pracy, samodzielności, skrupulatności,

- kreatywności i inicjatywy,

- biegłej znajomości programów: MS office, Excel,

- znajomości i umiejętności programowania obrabiarek CNC

$$
\text { Oferty prosimy składać na adres: }
$$

Bydgoskie Zakłady Sklejek "Sklejka-Multi" S.A.

85-752 Bydgoszcz, ul. Fordońska 154, lub e-mail: b.kasprzyk@sklejka.pl

\section{Niemieckojęzyczny Specjalista ds. Wsparcia Klienta \\ 1-26-21965/GP}

Niemieckojęzyczny Specjalista ds. Wsparcia Klienta 1-26-21965/GP office@grafton.pl Czy chciałbyś: powierzyć swój rozwój zawodowy jednej z największych firm z branży IT?

poznać bliżej środowisko multi-kulti?

otrzymać szanse rozwoju i nauki jaką daje nasz Klient?

Twoja praca bẹdzie polegać na wsparciu Klienta zagranicznego w rozwiązywaniu problemów, wyjaśnianiu watpliwości zwiazanych z obsługą komputera, codziennym kontakcie telefonicznym i e-mailowy z Klientami z Niemiec, Austrii, Szwajcarii, przyjmowaniu , przekazywaniu i monitorowaniu zgłoszeń użytkowników

WYMAGANIA

Zapraszamy osoby, które znają jẹzyk niemiecki na poziomie co najmniej dobrym i nie boją się go używać w codziennej komunikacj.

Jesteśmy otwarci zarówno na osoby z doświadczeniem jak i dopiero rozpoczynające swoją przygode z rynkiem pracy. Ważne jest abyś dobrze czuł siẹ w kontakcie z innymi, wykazywał siẹ cierpliwościa był otwarty na pracẹ w międzynarodowym zespole oraz naukę i szkolenia.

KORZYŚCI

Jeśli chcesz pracować w firmie, w której liczą się ludzie oraz która kolejny raz z rzẹdu znalazła siẹ w gronie najlepszych pracodawców ta oferta jest właśnie dla Ciebie! Firma inwestuje w rozwó] pracownikow, zapewnia im przyjazne srodowisko pracy, stabinnosc finansową oraz liczne szkolenia. Zatrudniony zostaniesz w oparciu o umowe o prace. Bejdziesz mógł korzystać m.in. z karty benefit, prywatnej opieki medycznej, ubezpieczenia na życie. Jeśli zdecydujesz się na relokację z innej miejscowości otrzymasz dofinansowanie relokacyjne.

Chẹtnie poznamy Ciẹ bliżej!

Jeżeli jesteś zainteresowany tą ofertą pracy prześlij do nas swoją aplikację wraz z numerem referencyjnym w tytule maila na adres: office@grafton.pl

Kontaktujemy siẹ tylko $z$ wybranymi osobami. Zaproszonych kandydatów rejestrujemy w naszej bazie danych. Nadesłanych dokumentów nie zwracamy. Gwarantujemy dyskrecję.

Prosimy o dopisanie klauzuli: "Zgadzam się na wykorzystanie przez firmę Grafton Recruitment Polska Sp. z o.0. $z$ siedzibą w Warszawie, ul. Sienna 39 danych osobowych wcelu pośrednictwa pracy (posiadania iprzetwarzania przekazanych danych osobowych oraz ich udosteppniania klientom wramach prowadzonych projektów rekrutacyjnych) zgodnie z obowiązująca Ustawa o Ochronie Danych Osobowych (Dz.U.2002 r. Nr $101 \mathrm{poz}$. 926) aż do momentu pisemnego wycofania zgody. Potwierdzam, że zostałem poinformowany o prawach do zmiany, dosteppu i ochrony tych danych".

ISO: 9001:2008 Certified Company

\section{Abbildung 9}

\subsection{Inkonsequente Stellenausschreibungen}

Die 15 inkonsequenten StAen aus dem deutschen Teilkorpus lassen sich in 2 Gruppen gliedern, in denen das generische Maskulinum (z. B. Bauleiter, Berufseinsteiger) neben geschlechtergerechten Formen im Begleittext (z. B. Bauingenieur/in) verwendet wird (Frauen werden diskriminiert) und geschlechtergerechte Formen (z. B. Haushälterin mit dem Zusatz "m/w") neben weiblichen Personenbezeichnungen (z. B. Nichtraucherin) benutzt werden (Männer werden diskriminiert). 
Als erfolgreiches mittelständisches Unternehmen in der Abbruch-, Erdund Entsorgungsbranche suchen wir zur weiteren Verstärkung unseres Teams für den Raum Südbayern

BAUINGENIEUR/IN (Dipl.-Ing.)

zur eingenständigen, kompletten Abwicklung von Bauvorhaben.

Wir suchen sowohl Berufseinsteiger als auch erfahrene Bauleiter auf diesem Gebiet und bieten Ihnen einen sicheren Arbeitsplatz mit

überdurchschnittlicher Bezahlung und gutem Betriebsklima.

Vollständige Bewerbungsunterlagen bitten wir zu richten an:

B. TRINKL GmbH \& Co. KG, Gautinger Str. 51, 82152 Krailling b. München oder per E-Mail an Herrn Janke: s.janke@trinklkg.de

stellenmarkt.sueddeutsche.de, 07.03. 2011
Wir, ein Industrieunternehmen im Einzugsbereich von Stuttgart, suchen für unser Gästehaus eine selbständig arbeitende

\section{Haushälterin $(\mathrm{m} / \mathrm{w})$}

die sich um den reibungslosen Ablauf des Hauses kümmert. Sie sollten über entsprechende Berufserfahrung in diesem Berufsfeld verfügen, eine gute Köchin sein und sich sehr gut organisieren können. Bei der Beschäffung von entsprechendem Wohnraum können wir Ihnen behilflich sein. Sie sollten nach Möglichkeit Nichtraucherin sein und einen Führerschein besitzen. Die Vergütung ist überdurchschnittlich hoch.

Zuschriften unter $\otimes$ ZS1891412.

stellenmarkt.sueddeutsche.de,12.03. 2011

Abbildung 10

32 inkonsequente StAen im polnischen Teilkorpus enthalten: (a) englische oder englische und polnische Stellenbezeichnungen, in denen man das generische Maskulinum benutzt und stellenweise auch neutrale Formen verwendet (z. B. osoby zainteresowane), um sich dann direkt an Männer zu wenden; b) Stellenbezeichnungen, mit denen man sich an Frauen wendet (z. B. recepcjonistka), daneben man aber auch maskuline (z. B. kandydaci) sowie neutrale Formen (z. B. osoby zainteresowane) benutzt. 

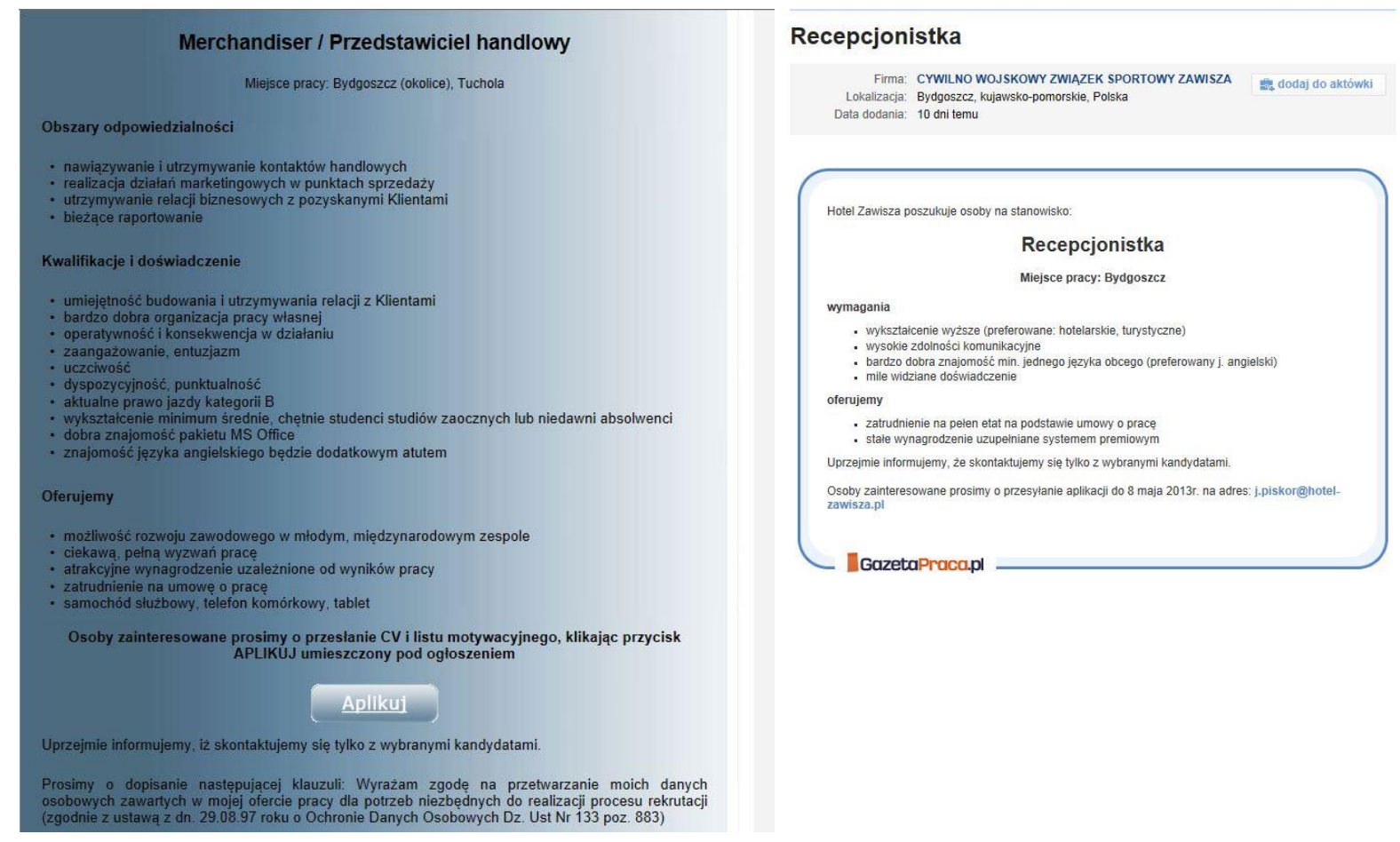

Abbildung 11

\section{$5 \quad$ Resümee}

Der Anteil der StAen im deutschen Teilkorpus, die sowohl Frauen als auch Männer ansprechen, d. h. geschlechtergerecht formuliert sind, beträgt $71 \%$; im gesamten polnischen Teilkorpus wurden dagegen keine geschlechtergerechten StAen gefunden. Diese Tatsache darf aber nicht interpretiert werden, dass es im Polnischen keine geschlechtergerechten StAen gibt, sondern dass feminine Berufsbezeichnungen sich in dieser Sphäre der Kommunikation (Online-Arbeitssuche) noch nicht durchgesetzt haben oder (was auch möglich ist) immer noch selten gebraucht werden und/oder nur schwer zu erfassen sind. Das erklärt aber, warum die Zahl geschlechtsspezifischer (14\%) und inkonsequenter StAen (15\%) im deutschen viel geringer ist als im polnischen Teilkorpus (68\% und 32\%). Allerdings sind in diesem Bereich für eine tragfähige Argumentation noch weitere umfangreichere Untersuchungen notwendig.

Aufgrund der analysierten Daten lässt sich schlussfolgern, dass sprachliche Gleichbehandlung beider Geschlechter im Deutschen mittlerweile Tatsache ist. Die jahrelange sprachkritische und -politische Arbeit der FeministInnen in Deutschland hat einen wichtigen Beitrag zur Herausbildung eines neuen Bewusstseins und zum laufenden Sprachwandel geleistet. Das feministische Bemühen um einen geschlechtergerechten Sprachgebrauch wurde und wird heute durch verschiedene Institutionen des gesellschaftlichen Lebens unterstützt, die rechtlich geregelte Richtlinien und Empfehlungen herausgegeben haben. Diese enthalten Hinweise für den täglichen Gebrauch und erleichtern das geschlechtergerechte Formulieren. In der polnischen Gender-Diskussion von heute werden andere Prioritäten gesetzt, die von verschiedenen politischen Gremien leider allzu oft für eigene Zwecke genutzt und ideologisch aufgeladen werden. Die geführte Diskussion liegt jedoch außerhalb der sprachpolitischen 
Sphäre, ohne die rechtliche Entscheidungen nicht möglich sind, wie die deutsche Praxis gezeigt hat. Dieser Weg muss in Polen noch gegangen werden.

\section{Literatur}

Ayaß, Ruth (2008): Kommunikation und Geschlecht. Eine Einführung. Stuttgart: Kohlhammer.

Anusiewicz, Janusz/Handke, Kwiryna (eds.) (1994): Płeć w języku i kulturze. Wrocław: Wydawnictwo Uniwersytetu Wrocławskiego. (= Język a kultura 9).

Arabski, Janusz/Ziębka, Justyna (eds.) (2010): Płeć języka - język płci. Katowice: Wydawnictwo Wyższej Szkoły Zarządzania Marketingowego i Języków Obcych. (= Językoznawstwo 7).

Belczyk-Kohl, Ivonne (2013): "Sprache und Geschlecht als Thema in der polnischen Sprachwissenschaft". In: Symanzik, Bernhard (ed.) (2013): Miscellanea Slavica Monasteriensia. Gedenkschrift für Gerhard Birkfellner, gewidmet von Freunden, Kollegen und Schülern. Berlin, LIT Verlag: 15-48.

Bochenek, Zofia (2010): Frauen in der Sprache. Analyse von ausgewählten polnischen und deutschen juristischen Dokumenten. Hamburg: Diplomica Verlag.

Braun, Friederike (2000): Mehr Frauen in die Sprache. Leitfaden zur geschlechtergerechten Formulierung. Kiel: Ministerium für Justiz, Frauen, Jugend und Familie des Landes Schleswig-Holstein.

Bulawka, Hanna Maria (2013): Gender Representations in the Polish Press. A Feminist Critical Discourse Study. Warszawa: Warszawska Firma Wydawnicza.

Bundesstelle für Büroorganisation und Bürotechnik (ed.) (2002): Sprachliche Gleichbehandlung von Frauen und Männern. Hinweise, Anwendungsmöglichkeiten und Beispiele. Köln. https://www.freiburg.de/pb/site/Freiburg/get/326311/Bundesverwaltungsamt_Merkblatt_Sprache_02.pdf, letzter Zugriff am 9. Februar 2015.

Chołuj, Bożena/Neusüß, Claudia (2004): "Geschlechtergleichstellung in Polen - zwischen historischem Erbe, kulturellen Eigenheiten und neuen Herausforderungen". In: Meuser, Michael/Neusüß, Claudia (eds.) (2004): Gender Mainstreaming. Konzepte Handlungsfelder - Instrumente. Bonn, Bundeszentrale für politische Bildung: 182-193.

Cieszkowski, Marek/Szczepaniak, Jacek (ed.) (2011): Język - rytuał -płeć. Bydgoszcz: Bydgoskie Towarzystwo Naukowe.

Engel, Ulrich (ed.) (2000): Deutsch-polnische kontrastive Grammatik. Bd. 1-2. Warszawa: Państwowe Wydawnictwo Naukowe.

Feigl, Susanne (2009): Geschlechtergerechte Stellenausschreibung. Wien: Bundesministerium für Frauen und öffentlichen Dienst. http://www.gleichbehandlungsanwaltschaft.at/DocView.axd?CobId=34384, letzter Zugriff am 9. Februar 2015.

Frank-Cyrus, Karin/Dietrich, Margot (1998): "Gesetze geschlechtergerecht gestalten - aber wie? Zwei Gutachten der Gesellschaft für deutsche Sprache für das Bundesministerium der Justiz". In: Schoenthal, Gisela (ed.) (1998): Feministische Linguistik - Linguistische Geschlechterforschung. Ergebnisse, Konsequenzen und Perspektiven. Hildesheim, Olms Verlag: 49-86.

Handke, Kwiryna (1986): "Rola kobiet w przekształcaniu współczesnej polszczyzny kolokwialnej". Rozprawy Komisji Językowej Łódzkiego Towarzystwa Naukowego XXXII: 101-107. 
Marek Cieszkowski: Zum geschlechtergerechten Sprachgebrauch am Beispiel deutscher und polnischer Stellenausschreibungen

Handke, Kwiryna (1989): "Styl kobiecy we współczesnej polszczyźnie kolokwialnej". Studia z Filologii Polskiej i Słowiańskiej XXVI: 5-24.

Handke, Kwiryna (1990): Wpływ emancypacji na język kobiet. In: Jedynak, Barbara (ed.) (1990): Kobieta w kulturze i społeczeństwie. Bd. 1. Lublin, Wydawnictwo Uniwersytet Marii Curie-Skłodowskiej: 156-170.

Handke, Kwiryna (1992): "Status rodzinny a zachowanie językowe kobiet i mężczyzn". Rocznik Naukowo-Dydaktyczny WSP w Krakowie. Prace językoznawcze 7: 151-160.

Handke, Kwiryna (1994a): "Przedmowa". In: Anusiewicz, Janusz/Handke, Kwiryna (eds.) (1994a): Płé́ w języku i kulturze. Wrocław, Wydawnictwo Uniwersytetu Wrocławskiego: 9-13. (= Język a kultura 9).

Handke, Kwiryna (1994b): "Język a determinanty płci". In: Anusiewicz, Janusz/Handke, Kwiryna (eds.) (1994b): Płeć w języku i kulturze. Wrocław, Wydawnictwo Uniwersytetu Wrocławskiego: 15-29. (= Język a kultura 9).

Hellinger, Marlis (1985): "Einführung". In: Hellinger, Marlis (ed.) (1985): Sprachwandel und feministische Sprachpolitik. Internationale Perspektiven. Opladen, Westdeutscher Verlag: 3-9.

Hellinger, Marlis (1990): Kontrastive feministische Linguistik. Mechanismen sprachlicher Diskriminierung im Englischen und Deutschen. Ismaning: Hueber Verlag.

Hellinger, Marlis/Bierbach, Christine (eds.) (1993): Eine Sprache für beide Geschlechter. Richtlinien für einen nicht-sexistischen Sprachgebrauch. Bonn: UNESCO.

Hellinger, Marlis (1997): "Der Diskurs der Verzerrung: Feministische Sprachpolitik und politische Korrektheit". Muttersprache 1/107: 35-46.

Homberger, Dietrich (1993): "Männersprache - Frauensprache: Ein Problem der Sprachkultur?" Muttersprache 1/103: 89-112.

Jaworski, Adam (1986): A linguistic picture of women's position in society. A Polish-English contrastive study. Frankfurt am Main/New York: Lang Verlag.

Karwatowska, Małgorzata (2004): "Językowe zachowania grzecznościowe a płeć (na podstawie wypowiedzi młodzieży szkół średnich". Poznańskie Spotkania Językoznawcze 12: 189-199.

Karwatowska, Małgorzata/Szpyra-Kozłowska, Jolanta (2005): Lingwistyka płci. Ona i on w języku polskim. Lublin: Wydawnictwo Uniwersytetu Marii Curie-Skłodowskiej.

Klann-Delius, Gisela (2005): Sprache und Geschlecht. Stuttgart: Metzler Verlag.

Łaziński, Marek (2006): O panach i paniach. Polskie rzeczowniki tytularne i ich asymetria rodzajowo-płciowa. Warszawa: Państwowe Wydawnictwo Naukowe.

Maciejewska, Anna (2012): Zum Gebrauch der femininen Formen am Beispiel der deutschen Stellenangebote. Bydgoszcz [Manuskript].

Miemietz, Bärbel (1993): Motivation zur Motion: zur Bezeichnung von Frauen durch Feminina und Maskulina im Polnischen. Frankfurt am Main etc.: Lang Verlag.

Miemietz, Bärbel (1996): "Ein marginales Phänomen? Zum Ausdruck des Sexus im Polnischen". In: Koschmal, Walter (ed.) (1996): Die Frau in der polnischen Gegenwartsliteratur. Köln etc.: Böhlau Verlag: 125-183.

Nowosad-Bakalarczyk, Marta (2003): "Czy język polski dyskryminuje kobiety?" Etnolingwistyka 15: 250-253.

Nowosad-Bakalarczyk, Marta (2009): Płeć a rodzaj gramatyczny we współczesnej polszczyźnie. Lublin: Uniwersytet Marii Curie-Skłodowskiej. 
Pusch, Luise F. (1984): Das Deutsche als Männersprache. Frankfurt am Main: Suhrkamp Verlag.

Pusch, Luise F. (1990): Alle Menschen werden Schwestern. Feministische Sprachkritik. Frankfurt am Main: Suhrkamp Verlag.

Samel, Ingrid (2000): Einführung in die feministische Sprachwissenschaft. Berlin: Schmidt Verlag.

Stalberg, Dagmar/Sczesny, Sabine (2001): "Effekte des generischen Maskulinums und alternativer Sprachformen auf den gedanklichen Einbezug von Frauen". Psychologische Rundschau 3/52: 131-140.

Trömel-Plötz, Senta (1984): Gewalt durch Sprache. Die Vergewaltigung von Frauen in Gesprächen. Frankfurt am Main: Fischer Verlag.

Werner, Martina (2007): Zur Verwendung geschlechtergerechter Sprache - die grammatische Kategorie Genus; http://www.google.pl/url?sa=t\&rct=j\&q=\&esrc=s\&frm=1\&source=web\&cd=1\&cad=rja\& ved $=0 \mathrm{CCcQFjAA \& url=http} \% 3 \mathrm{~A} \% 2 \mathrm{~F} \% 2 \mathrm{Fwww}$.frauenbeauftragte.unimuenchen.de $\% 2 \mathrm{Fber}$ ichte\%2Fberichte_veranstalt\%2Fhandreichung2007.pdf\&ei=P5kEU4WvOufhywPF24G4A Q\&usg=AFQjCNFo-aurVx-71uGSubJw5mAqA-B18Q; letzter Zugriff am 10.12.2013. 